Bryn Mawr College

Scholarship, Research, and Creative Work at Bryn Mawr

College

Physics Faculty Research and Scholarship

Physics

1977

\title{
The Haupt Effect: Coupled rotational and dipolar relaxation of methyl groups
}

Peter A. Beckmann

Bryn Mawr College, pbeckman@brynmawr.edu

S. Clough

J.W. Hennel

J.R. Hill

Let us know how access to this document benefits you.

Follow this and additional works at: https://repository.brynmawr.edu/physics_pubs

Part of the Physics Commons

\section{Custom Citation}

Beckmann, P., S. Clough, J. W. Hennel and J. R. Hill. 1977. "The Haupt Effect: Coupled rotational and dipolar relaxation of methyl groups." Journal of Physics C: Solid State Physics 0.5: 729-742.

This paper is posted at Scholarship, Research, and Creative Work at Bryn Mawr College. https://repository.brynmawr.edu/physics_pubs/138

For more information, please contact repository@brynmawr.edu. 


\title{
The Haupt effect: coupled rotational and dipolar relaxation of methyl groups
}

\author{
P Beckmann, S Clough, J W Hennel† and J R Hill \\ Department of Physics, University of Nottingham, University Park, Nottingham, UK
}

Received 23 June 1976 in final form 5 September 1976

\begin{abstract}
A theory is described for the dynamic proton dipolar polarization observed by Haupt in 4-methylpyridine following a sudden temperature change. The theory differs from that of Haupt in assuming that transitions which change the rotational quantum number of the 4-methyl group by \pm 3 occur very rapidly, maintaining thermal equilibrium within each of the three subsets of rotational levels corresponding to the three methyl group proton spin symmetry species $A, E^{a}$ and $E^{b}$. The difference of $A$ and $E$ species populations approaches the new equilibrium value slowly and exponentially, following the temperature jump, and generates dipolar polarization in the process. Transitions between $E^{a}$ and $E^{b}$ species lead to destruction of the polarization, whose evolution from zero due to these competing processes has the simple form $C[\exp (-a t)-\exp (-b t)]$. This is checked by a modified version of Haupt $s$ experiment in which the initial temperature jump is followed by a later burst of $R F$ pulses which reduces the dipolar polarization to zero. The subsequent evolution of the polarization always has the above form. The theory accounts for the dependence of $C$ on the temperature jump, and of $a$ and $b$ on the final temperature. The A to E conversion rate $a$ is obtained also by a calorimetric method which measures the rate at which rotational energy is transferred to the lattice. The result is in good agreement with the rate obtained from the dipolar polarization experiment.
\end{abstract}

\section{Introduction}

Haupt $(1972,1973)$ has discovered a very remarkable nuclear polarization effect shown by the protons in 4-methylpyridine at fairly low temperatures. The effect is connected with transitions between the rotational levels of the 4-methyl group. The rotation of this group is only weakly hindered and this is responsible for the unusual properties of this compound.

The polarization effect is easily observed at temperatures below about $50 \mathrm{~K}$ and in a magnetic field of a few thousand gauss. A rapid change of the sample temperature by a few degrees is followed during the next few minutes by the growth of a very large proton dipolar polarization. This means that the nuclear moments become partially oriented relative to the dipolar fields of neighbouring protons, though there is little change in the degree of orientation relative to the external magnetic field (Zeeman polarization). After reaching a maximum value, the dipolar polarization decays almost to zero, since the

$\uparrow$ On leave in 1975 from the Institute of Nuclear Physics, Krakow, Poland. 
equilibrium dipolar polarization is very small. The maximum dipolar polarization observed is about $10^{4}$ times larger than this equilibrium value (Haupt 1973). The time dependence of the additional dipolar polarization following the temperature jump is well described by

$$
P_{\mathrm{D}}(t)=C[\exp (-a t)-\exp (-b t)]
$$

where $C$ depends on both initial and final temperatures, and $a$ and $b$ depend only on the final temperature. This was established by Haupt (1973) who also measured the temperature dependence of the rate constants $a$ and $b$ and the enhancement factor $C$.

Two rather different theoretical models have been used to account for the polarization, namely Haupt (1972) and Clough and Hill (1974). We shall discuss later in more detail the differences between these theories, but the essential feature is that the latter theory uses fewer parameters to describe the populations of the levels of the methyl group. As a result of this relative simplicity, more detailed predictions are possible and in particular, a pair of coupled differential equations are obtained leading to an expression in the form of equation (1). A defect of the simpler theory though is that it describes the methyl group in terms of a tunnelling harmonic oscillator, assuming moderately strong hindering of the rotation, while recent nuclear spin-lattice relaxation measurements by Zweers et al (1974) and neutron scattering studies by Alefeld et al (1975) have demonstrated that the 4-methyl group in 4-methylpyridine is more properly described in terms of a free rotor. One object of this paper is to remedy this defect while preserving the simple result (1). The experiments reported in this paper provide a more stringent test of the theory than is offered simply by observing that equation (1) is obeyed following a temperature jump. In a slight elaboration of the Haupt experiment, the temperature jump is followed by one or more bursts of radiofrequency pulses, and the evolution of $P_{\mathrm{D}}(t)$ is followed after the pulses. These measurements on the spin system are supplemented by a second experiment in which the slow transfer of rotational energy to the lattice vibrations is observed by thermal measurements.

The essential theoretical idea may be described as a single temperature assumption for the populations of the rotational levels. The experiments may be regarded as a test of this assumption. They provide strong evidence of its validity. A second consequence of the experiments is that they lead to more accurate values of the parameters $a$ and $b$, since no measurements need to be made immediately following the temperature jump when the sample temperature may not be uniform. The range of rate constant which can be measured is also extended.

Although there is normally no macroscopic magnetic moment associated with dipolar polarization, it is easily measured using a pulsed nuclear magnetic resonance spectrometer. A short resonant RF pulse induces a transient macroscopic magnetic moment perpendicular to the applied magnetic field. By observing the resulting signal using a phase sensitive detector, the contributions which arise from dipolar and Zeeman polarizations may be separated by appropriate choice of the reference phase, and both may be measured independently. If the amplitude of the inspection pulse is small, the dipolar polarization may be measured without significantly changing its value. Repeated inspections then allow the evolution of $P_{\mathrm{D}}(t)$ to be followed.

\section{Theoretical discussion}

The most important difference between the torsional oscillator and free rotor models of 
the methyl group motion is that while only the lowest torsional oscillator level would be populated substantially at the temperature of the experiments, several free rotor levels have to be taken into account. The important point is that the differential equation for $P_{\mathrm{D}}(t)$ is coupled to the differential equations for the parameters which describe the deviation of the rotor populations from thermal equilibrium values. In the theory of Haupt (1972) this would be $(n-1)$ parameters for $n$ levels. It is clear that the simplicity of the result (1) obtained by Clough and Hill (1974) can only be preserved in the free rotor model if no additional parameters are introduced to describe the additional populated levels.

A characteristic feature of the thermodynamic approach to the theory of magnetic resonance (Jeener 1968, Goldman 1970) is the distinction between slow and fast transitions. The former do not appear explicitly in the theoretical development, but are assumed to maintain the system in a state of quasi-equilibrium, enabling the density matrix to be written in terms of a few parameters. The rate constants for the evolution of these parameters are then evaluated from appropriately weighted sums of the slow transition probabilities. In the present case of transitions between methyl group rotational states, we may distinguish two kinds of transition, those in which the rotational quantum number changes by some multiple of three, and those in which the change of the rotational quantum number is not a multiple of three. The former involve no change in the proton spin state of the methyl group while the latter involve such a change. We shall therefore regard the former as fast and the latter as slow.

The wavefunctions for a free rotor having a single rotational degree of freedom with coordinate $\phi$ have the form $\exp (\mathrm{i} m \phi$ ) where $m=0, \pm 1, \pm 2$, etc. If $P$ is an operator which cyclically permutes the space coordinates of the three protons of the methyl group, then $P \exp (\mathrm{im} \phi)=\exp (\mathrm{im}(\phi+2 \pi / 3))$. Thus the rotational functions are eigenfunctions of $P$ with eigenvalues $\exp (2 m i \pi / 3)$. If $P_{S}$ is an operator which cyclically permutes the spin states of the three protons, then the nuclear spin function is an eigenfunction of $P_{\mathrm{S}}$ with eigenvalue $\exp (-2 m i \pi / 3)$ since the indistinguishability of the protons requires that the total state function be invariant under the operator $P P_{\mathrm{S}}$ which permutes both space and spin coordinates. Thus the rotational states with $m=0, \pm 3, \pm 6 \ldots$ are associated with proton spin functions of type A (eigenvalue of $P_{\mathbf{S}}=1$ ), those having $m=-4,-1,2,5$, etc. with functions of type $\mathrm{E}^{\mathrm{b}}$ (eigenvalue of $P_{\mathrm{S}}=\epsilon=\exp (2 \pi \mathrm{i} / 3)$ and those having $m=-2,1,4$, etc. with functions of type $\mathrm{E}^{\mathrm{a}}$ (eigenvalue of $P_{\mathrm{S}}=\epsilon^{*}$ ).

In thermal equilibrium with the lattice at temperature $\theta^{\prime}$ the populations of the rotational levels of a single rotor are proportional to $\exp \left(-h v_{\mathrm{t}} m^{2} /\left(k \theta^{\prime}\right)\right)$ where $h v_{\mathrm{t}}$ is the splitting between the lowest pair of levels. When the lattice temperature changes suddenly to $\theta$ the populations of the rotational levels approach the new equilibrium values in two stages. The fast transitions rapidly establish thermal equilibrium within the A type levels $m=0, \pm 3, \pm 6$, etc. and also within the $\mathrm{E}$ type levels. The second slow stage involves conversion between the two types. Following the temperature jump the populations rapidly become proportional to $\exp \left(-h v_{1} m^{2}(k \theta)\right) \exp (\beta x)$ where $x=1$ for A levels and -1 for $E$ levels. Then in the second stage, the parameter $\beta$ falls to zero. The initial value $\beta_{0}$ after the temperature jump can be found by requiring that the average value of $x$ does not change during the temperature jump.

$$
\begin{aligned}
\sum_{m=-\infty}^{\infty} x \mu_{m} \exp \left[-h v_{\mathrm{t}} m^{2} /\left(k \theta^{\prime}\right)\right] / Z\left(\theta^{\prime}\right)= \\
\sum_{m=-\infty}^{\infty} x \mu_{m} \exp \left[-h v_{\mathrm{t}} m^{2} /(k \theta)\right] \exp \left(\beta_{0} x\right) / Z(\theta)
\end{aligned}
$$


where $\mu_{m}$ is the nuclear spin multiplicity ( 4 for $m=0 \pm 3$ etc; otherwise 2 ) and

$$
\begin{aligned}
& Z(\theta)=\sum_{m=-\infty}^{\infty} \mu_{m} \exp \left[-h v_{\mathrm{t}} m^{2} /(k \theta)\right] \exp \left(\beta_{0} x\right) \\
& Z\left(\theta^{\prime}\right)=\sum_{m=-\infty}^{\infty} \mu_{m} \exp \left[-h v_{\mathrm{t}} m^{2} /\left(k \theta^{\prime}\right)\right] .
\end{aligned}
$$

It is convenient to perform the summations separately over the $A$ and the $E$ levels, so we define $Z_{\mathrm{A}}(\theta)$ and $Z_{\mathrm{E}}(\theta)$ as follows :

$$
\begin{aligned}
& Z_{\mathrm{A}}(\theta)=\sum_{m=0, \pm 3, \pm 6,} \mu_{m} \exp \left[-h v_{\mathrm{t}} m^{2} /(k \theta)\right] \\
& Z_{\mathrm{E}}(\theta)=\sum_{m= \pm 1, \pm 2, \pm 4,} \mu_{m} \exp \left[-h v_{\mathrm{t}} m^{2} /(k \theta)\right] .
\end{aligned}
$$

Then $Z(\theta)=Z_{\mathrm{A}}(\theta) \exp \left(\beta_{0}\right)+Z_{\mathrm{E}}(\theta) \exp \left(-\beta_{0}\right)$ and one easily finds from equation (2) that $\beta_{0}=\frac{1}{2} \ln \left(Z_{\mathrm{E}}(\theta) Z_{\mathrm{A}}\left(\theta^{\prime}\right)\right)-\frac{1}{2} \ln \left(Z_{\mathrm{A}}(\theta) Z_{\mathrm{E}}\left(\theta^{\prime}\right)\right)$. A related and physically more important quantity is the average value of $x$, since it is the change in the equilibrium value of this parameter which determines how many methyl groups convert from A type to E type or vice versa as a consequence of the temperature jump. We should therefore expect that the dynamic polarization is proportional to this change and therefore proportional to the change in $\left(Z_{\mathrm{A}}-Z_{\mathrm{E}}\right) /\left(Z_{\mathrm{A}}+Z_{\mathrm{E}}\right)$. Now Haupt has obtained an empirical function from the results of many experiments over a wide temperature range, which gives the maximum polarization obtained from a temperature jump from the difference in the value of the function at the two temperatures. Figure 1 shows a comparison between the results of

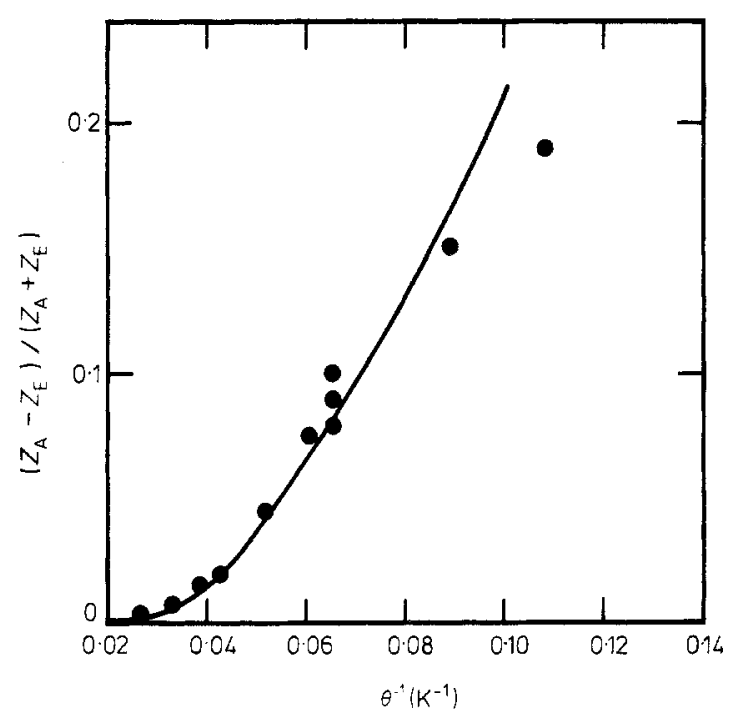

Figure 1. The evidence that the dipolar polarization is generated during $A$ to $E$ nuclear spin symmetry conversion. The experimental points are those of Haupt (1973) showing the magnitude of the dipolar polarization ( $C$ in equation (1)) in arbitrary units for a jump from high temperature to temperature $\theta$. The curve is the fractional difference in the equilibrium populations of $\mathrm{A}$ and $\mathrm{E}$ spin species for a nearly freely rotating methyl group. 
Haupt (1973, figure 11) with the function $c\left(Z_{\mathrm{A}}-Z_{\mathrm{E}}\right) /\left(Z_{\mathrm{A}}+Z_{\mathrm{E}}\right)$. This was calculated using the energy splittings measured by Alefeld et al (1975), the first and second excited rotational levels being $6.0 \mathrm{~K}$ and $21 \mathrm{~K}$ above the ground state. The third rotational level was taken to be $50 \mathrm{~K}$ above the ground state and higher levels $7 n^{2} \mathrm{~K}$ where $n=4$, 5, etc. In the temperature range of interest, these higher levels are unimportant. The general agreement shown by figure 1 is good, except at the lowest temperature where the experimental data are less certain because the normalization procedure used by Haupt (1973) depends on accurate measurements of the rate constants $a$ and $b$ which are difficult to obtain at low temperatures when $b$ becomes large. No dipolar polarization is expected for a temperature jump above $50 \mathrm{~K}$, since above this temperature $Z_{\mathrm{A}}$ and $Z_{\mathrm{E}}$ are essentially equal and no changes in the $A$ and $E$ populations result from the jump.

Three other questions arise in connection with the Haupt effect. They are (i) the mechanism of the dynamic polarization, (ii) the origin of equation (1), and (iii) the temperature dependence of $a$ and $b$. Before embarking on the detailed theory we shall

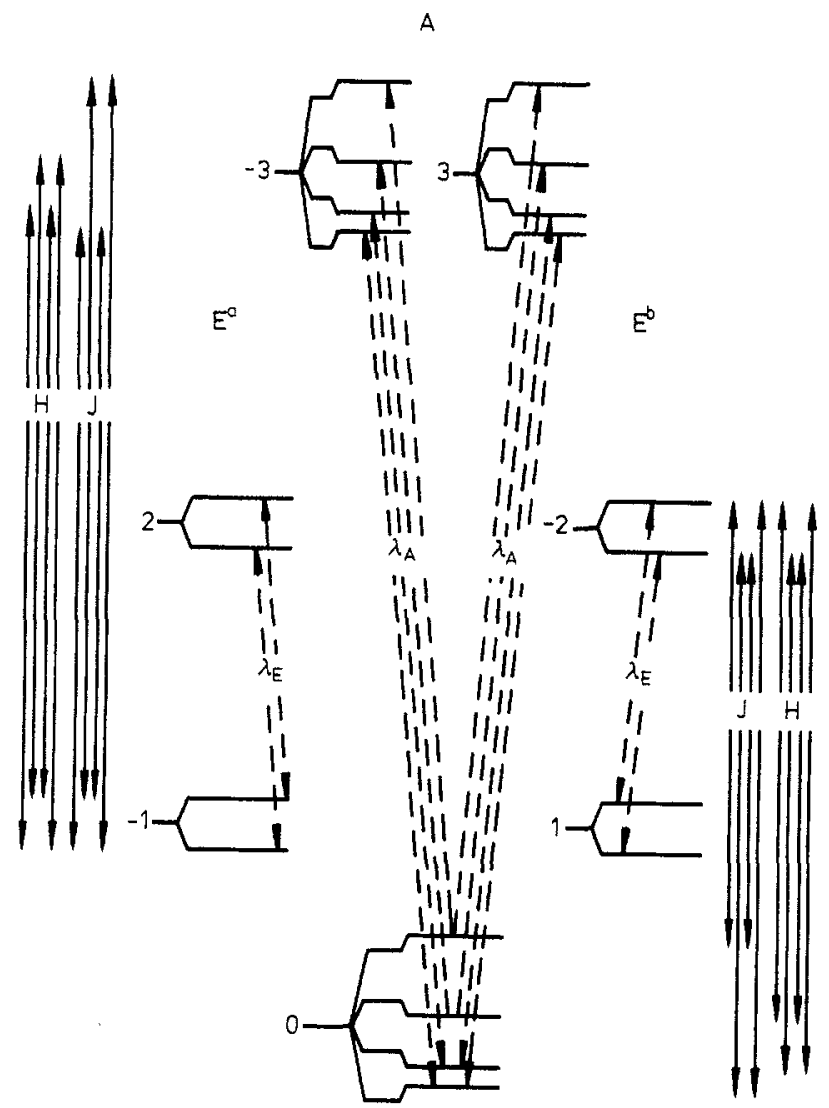

Figure 2. The lowest seven rotational levels of a nearly freely rotating methyl group. The levels are arranged in three columns corresponding to the nuclear spin species. Fast spin-independent transitions indicated by broken lines maintain thermal equilibrium in each column. The allowed $\mathrm{A}$ to $\mathrm{E}$ transitions which cause dipolar polarization are shown by continuous lines and divided into groups $\mathrm{H}$ and $\mathrm{J}$ which generate polarization of opposite signs. $\mathrm{E}^{\mathrm{a}}$ to $\mathrm{E}^{\mathrm{b}}$ transitions especially between rotational levels \pm 1 are responsible for relaxation of the polarization. 
indicate the answers to these questions. Figure 2 shows schematically all the magnetic sublevels of the lowest seven rotational levels for a single methyl group. The allowed A to $\mathrm{E}$ transitions are indicated at the sides of the figure, collected in two groups labelled $\mathrm{H}$ and $\mathrm{J}$. The change in the dipolar energy is equal in magnitude but opposite in sign for the $\mathrm{H}$ and $\mathrm{J}$ transitions, but the sum of the transition probabilities weighted with the changes in dipolar energy is not zero, even when averaged over all orientations of methyl groups. This results in a change in the dipolar energy when $A$ to $E$ conversion occurs. We may describe $A$ to $E$ transitions as polarizing transitions. $E^{a}$ to $E^{b}$ conversion does not have this property and can only destroy the polarization. Following a temperature jump, the dipolar polarization builds up until the rate at which it is destroyed by $E^{a}$ to $E^{b}$ transitions is equal to the rate at which it is created by $A$ to $E$ conversion. The polarization subsequently falls with the rate of $A$ to $E$ conversion. This accounts for the form of equation (1) and identifies $b$ as the $\mathrm{E}^{\mathrm{a}}$ to $\mathrm{E}^{\mathrm{b}}$ conversion rate. The temperature dependence of this parameter is quite different from that of $a$ because $\mathrm{E}^{\mathrm{a}}$ to $\mathrm{E}^{\mathrm{b}}$ conversion involves no exchange of energy with the lattice vibrations, and so becomes faster with falling temperature while $\mathrm{A}$ to $\mathrm{E}$ conversion becomes slower.

\section{The coupled relaxation equations in the high-temperature approximation}

In this section we shall give a theory of the Haupt effect appropriate at the relatively high temperatures where the polarization is small. This permits an approximation which greatly simplifies the analysis, while exhibiting the essential features of the theoretical argument. It also provides a framework for a more detailed treatment discussed in the next section.

We consider a sample containing many methyl groups and many other protons not in groups. The Hamiltonian is written

$$
\mathscr{H}=\mathscr{H}_{\mathrm{R}}+\mathscr{H}_{\mathrm{Z}}+\mathscr{H}_{\mathrm{D}}
$$

where the terms represent the rotational energy of the methyl groups, the Zeeman energy of the proton magnetic moments in the external magnetic field, and the proton dipole-dipole interactions. We also introduce the operators $Q_{k}=\frac{2}{3}\left(P_{k}+P_{k}^{*}-\frac{1}{2}\right)$, where $P_{k}$ is the permutation operator for methyl group $k$. The parameter $x$ introduced in the last section is the eigenvalue of $Q_{k}$.

The dipolar Hamiltonian $\mathscr{H}_{\mathrm{D}}$ is decomposed in two ways. First it is separated as usual into a secular and a non-secular part, the former commuting with $\mathscr{H}_{\mathrm{Z}}$. Then from this secular part is extracted a part $\mathscr{H}_{\mathrm{D}}^{0}$ which is invariant under any of the operators $Q_{k}$.

$$
\mathscr{H}_{\mathrm{D}}=\mathscr{H}_{\mathrm{D}}^{0}+\mathscr{H}_{\mathrm{d}}
$$

We assume that rapid transitions among the rotational levels and nuclear spin flip-flops result in the establishment of a quasi-equilibrium density matrix with the form

$\rho=\exp \left[-\mathscr{H}_{\mathrm{R}} /(k \theta)\right] \exp \left(\beta \sum_{k} Q_{k}\right) \exp \left[-\mathscr{H}_{\mathrm{Z}} /\left(k \theta_{\mathrm{Z}}\right)\right] \exp \left[-\mathscr{H}_{\mathrm{D}}^{0} /\left(k \theta_{\mathrm{D}}\right)\right] / Z$

where $Z$ normalizes $\rho$ so that $\operatorname{Tr} \rho=1$. We have introduced the Zeeman and dipolar temperatures $\theta_{\mathrm{Z}}$ and $\theta_{\mathrm{D}}$. It is important that the four operators in equation (6) commute with each other if (6) is to represent a quasi-equilibrium state; this is the reason for the decomposition (5). In this section we restrict ourselves to small values of $\beta$, and use the 
usual high-temperature approximation for the nuclear spin terms to obtain

$$
\begin{aligned}
& \rho \simeq \exp \left[-\mathscr{H}_{\mathrm{R}} /(k \theta)\right]\left[1+\beta \sum_{k} Q_{k}-\mathscr{H}_{\mathrm{Z}}\left(k \theta_{\mathrm{Z}}\right)^{-1}-\mathscr{H}_{\mathrm{D}}^{0}\left(k \theta_{\mathrm{D}}\right)^{-1}\right] / Z \\
& Z \simeq \operatorname{Tr}\left(\exp \left[-\mathscr{H}_{\mathrm{R}} /(k \theta)\right]\right) .
\end{aligned}
$$

The deviation of the density matrix from thermal equilibrium which is characterized by $\beta=0$ and $\theta_{\mathrm{Z}}=\theta_{\mathrm{D}}=\theta$ is

$$
\rho-\rho_{\text {eq }}=\rho_{\mathrm{R}}\left(\beta \sum_{k} Q_{k}+\beta_{\mathrm{Z}} \mathscr{H}_{\mathbf{z}}+\beta_{\mathrm{D}} \mathscr{H}_{\mathrm{D}}^{0}\right)
$$

where

$$
\begin{aligned}
& \rho_{\mathrm{R}}=\exp \left[-\mathscr{H}_{\mathrm{R}} /(k \theta)\right] / Z \\
& \beta_{\mathrm{Z}}=(k \theta)^{-1}-\left(k \theta_{\mathrm{Z}}\right)^{-1} \\
& \beta_{\mathrm{D}}=(k \theta)^{-1}-\left(k \theta_{\mathrm{D}}\right)^{-1} .
\end{aligned}
$$

Differential equations for the three parameters $\beta, \beta_{\mathrm{Z}}, \beta_{\mathrm{D}}$ may then be obtained using timedependent perturbation theory. The perturbation is that part of $\mathscr{H}_{\mathrm{d}}$ which is modulated by random rotations of the methyl groups occurring as a result of interaction of the rotors with the thermal vibrations of the crystal lattice. We again decompose $\mathscr{H}_{\mathrm{d}}$ into a part $\mathscr{H}_{\mathrm{d}}^{\mathrm{A}}$ which is invariant under any of the operators $Q_{k}$ and a part $\mathscr{H}_{\mathrm{d}}^{\mathrm{E}}$ which is not. The latter part contains terms which fluctuate at rates related to the rate of lattice-induced transitions which interrupt the rotational motion of the methyl groups (Clough 1976). Then time-dependent perturbation theory may be used, following Look and Lowe (1966) to obtain a rate equation for the expectation value $\left\langle\mathscr{H}_{\mathrm{D}}^{0}\right\rangle$ of the dipolar energy.

$$
\mathrm{d}\left\langle\mathscr{H}_{\mathrm{D}}^{0}\right\rangle / \mathrm{d} t=-\operatorname{Tr}\left\{\mathscr{H}_{\mathrm{D}}^{0} \int_{0}^{\infty}\left\langle\left[\mathscr{H}_{\mathrm{d}}^{\mathrm{E}}(0),\left[\mathscr{H}_{\mathrm{d}}^{\mathrm{E}}(t),\left(\rho-\rho_{\text {eq }}\right)\right]\right]\right\rangle \mathrm{d} t\right\} .
$$

In equation (10) it is understood that $\mathscr{H}_{\mathrm{d}}^{\mathrm{E}}$ is transformed into the interaction representation, part of the time dependence coming from that, and part coming from the lattice induced transitions previously mentioned. The angular brackets on the right hand side of equation (10) indicate an average over the random part of the time dependence, introducing the correlation functions for the various terms. Further details may be found in a discussion of Zeeman relaxation (Clough 1976) which parallels the present one.

From equation (9) we may also find that $\mathrm{d}\left\langle\mathscr{H}_{\mathrm{D}}^{0}\right\rangle / \mathrm{d} t=\operatorname{Tr}\left(\rho_{\mathrm{R}}\left(\mathscr{H}_{\mathrm{D}}^{0}\right)^{2}\right) \mathrm{d} \beta_{\mathrm{D}} / \mathrm{d} t$. a result which, combined with equation (10) gives an expression for $\mathrm{d} \beta_{\mathrm{D}} / \mathrm{d} t$. Similar expressions may be obtained for $\mathrm{d} \beta / \mathrm{d} t$ and $\mathrm{d} \beta_{\mathrm{Z}} / \mathrm{d} t$ by evaluating the rate of change of the expectation values of the operators $\sum_{k} Q_{k}$ and $\mathscr{H}_{\mathrm{Z}}$. The result has the form

$$
\frac{\mathrm{d}}{\mathrm{d} t}\left[\begin{array}{l}
\beta_{\mathrm{D}} \\
\beta \\
\beta_{\mathrm{Z}}
\end{array}\right]=-\left[\begin{array}{lll}
U & W & 0 \\
X & V & 0 \\
0 & 0 & Y
\end{array}\right]\left[\begin{array}{l}
\beta_{\mathrm{D}} \\
\beta \\
\beta_{\mathrm{Z}}
\end{array}\right]
$$

The coefficient $X$ for example is given by

$$
X=\operatorname{Tr}\left\{\sum_{k} Q_{k} \int_{0}^{\infty}\left\langle\left[\mathscr{H}_{\mathrm{d}}^{\mathrm{E}}(0),\left[\mathscr{H}_{\mathrm{d}}^{\mathrm{E}}(t), \rho_{\mathrm{R}} \mathscr{H}_{\mathrm{D}}^{0}\right]\right]\right\rangle \mathrm{d} t\right\} / \operatorname{Tr}\left(\rho_{\mathrm{R}}\left(\sum_{k} Q_{k}\right)^{2}\right) .
$$

The detailed evaluation of the coefficients in equation (11) is a formidable task, though often dominant terms may be identified and their values calculated without too much trouble. In the present case the dipole-dipole interactions within a methyl group are 
much more important than interactions between the protons of methyl groups and other protons, and in a later section we shall introduce explicitly the contributions from these most important dipolar terms. Previous discussions of the Haupt effect have considered only these intramethyl group interactions. Here we include, at least formally, all interactions.

The terms coupling $\beta_{\mathrm{Z}}$ to $\beta$ and $\beta_{\mathrm{D}}$ vanish because the corresponding traces are zero. For this reason we can ignore the Zeeman polarization. It is in fact coupled to the rotational polarization (Emid et al 1974) and detailed expressions for the rate constants have been given elsewhere (Clough 1976). The main concern of the present paper is whether equation (11) correctly describes the time dependence of $\beta_{\mathrm{D}}$ which being proportional to $\left\langle\mathscr{H}_{\mathrm{D}}\right\rangle$ may, in the present approximation, be identified with $P_{\mathrm{D}}$ in equation(1). Following the sudden change of temperature at time $t=0$, one has $\beta=\beta_{0}, \beta_{\mathrm{D}}=0$. Integration of (11) then gives (1) with $P_{\mathrm{D}}=\beta_{\mathrm{D}}$ and $C=W \beta_{0} /(a-b)$, the rate constants $a$ and $b$ being $\frac{1}{2}(U+V) \pm\left(\frac{1}{4}(U-V)^{2}+W X\right)^{1 / 2}$. Thus equation (11) is consistent with the results of Haupt. We shall describe further experiments to test equation (11), but first we shall mention analogous equations which apply when $\beta$ is not small.

\section{The coupled relaxation equations at low temperature}

From figure 1 we may find that a temperature jump between 10 and $25 \mathrm{~K}$ results in an initial value $\beta_{0}$ of $\pm \frac{1}{2} \ln (1 \cdot 35)$, the sign depending on the direction of the jump. Clearly the assumption that $\beta$ is small is not very satisfactory, at least for the early stages of the evolution of the dipolar polarization. When $\beta$ is not small the derivation of coupled equations analagous to (11) is very much more complicated. The result though is similar to (11) except that small non-linear terms arise and the dynamical coordinate to which $P_{\mathrm{D}}$ is coupled is not $\beta$, but $\sigma$ where

$$
\sigma=\frac{2 Z_{\mathrm{A}} Z_{\mathrm{E}}(\exp (\beta)-\exp (-\beta))}{\left(Z_{\mathrm{A}}+Z_{\mathrm{E}}\right)\left(Z_{\mathrm{A}} \exp (\beta)+Z_{\mathrm{E}} \exp (-\beta)\right)} .
$$

This is the deviation of the fractional difference of $A$ and $E$ populations from the thermal equilibrium value, the obvious driving force of the dipolar polarization. The rather formidable theory will not be given here since the main conclusions will be obtained by rather simple arguments in the next section. We simply assume that equation (11) is generally valid if $\beta_{\mathrm{D}}$ is replaced by $P_{\mathrm{D}}$ and $\beta$ by $\sigma$.

\section{The temperature dependence of the spin symmetry conversion rates}

We have accounted for the form of equation (1) through the coupled equations (11) and the magnitude of $C$ as shown in figure 1 . There remains the question of the temperature dependence of the rate constants $a$ and $b$. Over much of the temperature range the ratio $a / b$ is large and we may identify $a$ with $U$ and $b$ with $V$. Intramethyl group dipolar interactions make comparable contributions to $U$ and $V$, so the difference between the two must be due to interactions with other protons. These interactions introduce new transitions of the methyl group, namely between $\mathrm{E}^{\mathrm{a}}$ and $\mathrm{E}^{\mathrm{b}}$ species. These make no contribution to $\mathrm{V}$ because they cause no change in $\sigma$, but they do contribute to the dipolar relaxation rate $U$. Of all possible $\mathrm{E}^{\mathrm{a}}$ to $\mathrm{E}^{\mathrm{b}}$ transitions, one type has a special importance. These are the 
transitions between degenerate rotational levels, involving a change in the sign of the rotational quantum number. Because there is no exchange of energy with the lattice they have a temperature dependence quite different from other transitions and they are responsible for the difference in the temperature dependence of $U$ and $V$ which is shown in figure 3.
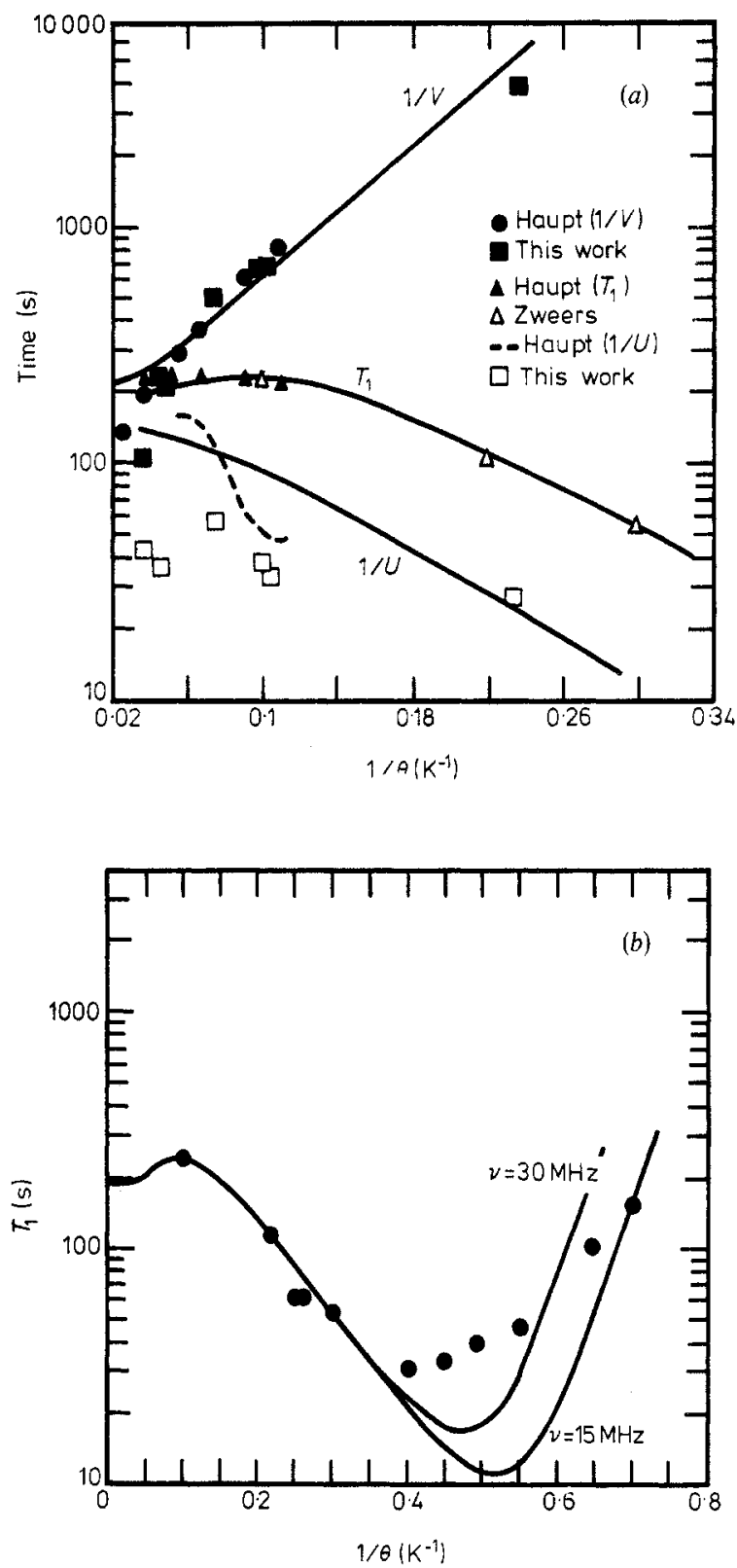

Figure 3. The temperature dependence of the two time constants of the dipolar polarization and of the nuclear Zeeman relaxation time $T_{1}$. The curves are calculated from equations (18-20) with parameters given in the text. Figure $3(b)$ extends the $T_{1}$ curves down to $1.5 \mathrm{~K}$ and shows the data of Zweers et al (1974). 
Turning first to the temperature dependence of $V$, we introduce $T_{i j}^{\mathrm{AE}}$, the transition probability from an A state $i$ of energy $W_{i}^{\mathrm{A}}$ to an $E$ state $j$ of energy $W_{j}^{\mathrm{E}} . T_{j i}^{\mathrm{EA}}$ is the probability of the reverse transition:

$$
T_{j i}^{\mathrm{EA}} \exp \left[-W_{j}^{\mathrm{E}} /(k \theta)\right]=T_{i j}^{\mathrm{AE}} \exp \left[-W_{i}^{\mathrm{A}} /(k \theta)\right] .
$$

Then $V$ may be written

$$
V=\sum_{i}^{\mathrm{A}} \sum_{j}^{\mathrm{E}}\left\{T_{i j}^{\mathrm{AE}} Z_{\mathrm{A}}^{-1} \exp \left[-W_{i}^{\mathrm{A}} /(k \theta)\right]+T_{j i}^{\mathrm{EA}} Z_{\mathrm{E}}^{-1} \exp \left[-W_{j}^{\mathrm{E}} /(k \theta)\right]\right\} .
$$

This is just the sum of all transitions from $A$ states to $E$ states and vice versa, each weighted with the population factor of the initial state. This is a simple generalization of the well known result for a two-level system that the relaxation rate of the population difference is the sum of the two transition rates between the levels. The sums $\sum_{i}^{A}$ and $\sum_{j}^{E}$ run respectively over all $A$ and all $E$ states. Using equation (13) we can rewrite (14)

$$
V=\left(Z_{\mathrm{A}}^{-1}+Z_{\mathbf{E}}^{-1}\right) \sum_{i}^{\mathrm{A}} \sum_{j}^{\mathrm{E}} T_{i j}^{\mathrm{d}} \exp \left[-W_{i j}^{\mathrm{g}} /(k \theta)\right]
$$

where $T_{i j}^{\mathrm{d}}$ is the downward transition probability, i.e. the greater of the two transition probabilities $T_{i j}^{\mathrm{AE}}$ and $T_{j i}^{\mathrm{EA}}$ which involves the lattice in absorption rather than emission of energy. $W_{i j}^{\mathrm{g}}$ is the greater of the two energies $W_{i}^{\mathrm{A}}$ and $W_{j}^{\mathrm{E}}$. We assume that $T_{i j}^{\mathrm{d}}$ is givul1 by the usual result of time-dependent perturbation theory:

$$
T_{i j}^{\mathrm{d}}=\hbar^{-2} D_{i j}^{2} \tau_{i j} /\left(1+\hbar^{-2} W_{i j}^{2} \tau_{i j}^{2}\right)
$$

where $W_{i j}=W_{i}-W_{j}$ and $\tau_{i j}$ is the correlation time for the dipole-dipole matrix element $D_{i j}$ and is determined by the lifetimes of the two states $\tau_{i}$ and $\tau_{j}$.

$$
\tau_{i j}^{-1}=\tau_{i}^{-1}+\tau_{j}^{-1}
$$

Thus the spin symmetry conversion transitions occur due to modulation of dipolar matrix elements as a result of the fast spin-independent transitions.

We may now give a simple discussion of the temperature dependence of $V$ in terms of the levels and transitions shown in figure 2. The fast downward transition rates $\lambda_{\mathrm{E}}$ and $\lambda_{\mathrm{A}}$ are assumed to be temperature-independent, with the upward rates smaller by the Boltzmann factor. Thus the lifetimes of the \pm 3 levels are equal to $\lambda_{\mathrm{A}}^{-1}$ and the lifetimes of the \pm 2 levels are $\lambda_{\mathrm{E}}^{-1}$. The others are given by $\tau_{1}=\tau_{-1}=\lambda_{\mathrm{E}}^{-1} \exp \left[W_{21} /(k \theta)\right]$ and $\tau_{0}=$ $\frac{1}{2} \lambda_{\mathrm{A}}^{-1} \exp \left[W_{30} /(k \theta)\right]$. Using these with (16) and (17) in (15) and neglecting the 1 in the denominator of $(16)$, we obtain

$$
\begin{gathered}
V=24 \Delta^{2}\left(Z_{\mathrm{A}}^{-1}+Z_{\mathrm{E}}^{-1}\right)\left(W_{20}^{-2}\left\{\lambda_{\mathrm{E}}+2 \lambda_{\mathrm{A}} \exp \left[-W_{30} /(k \theta)\right]\right\} \exp \left[-W_{20} /(k \theta)\right]\right. \\
\left.+W_{31}^{-2}\left\{\lambda_{\mathrm{A}}+\lambda_{\mathrm{E}} \exp \left[-W_{21} /(k \theta)\right]\right\} \exp \left[-W_{30} /(k \theta)\right]\right)
\end{gathered}
$$

We have included here the explicit value of the sum of the intramethyl group dipole-dipole matrix elements over all the magnetic sublevels, introducing $\Delta=3 \hbar^{2} \gamma^{2} /\left(8 r^{3}\right)$ where $r$ is the interproton distance. If equation (18) is compared with the observed temperature dependence of the rate constant $a$, it becomes clear that the dominant term below $20 \mathrm{~K}$ is the one involving $\lambda_{\mathrm{E}} \exp \left[-W_{20} /(k \theta)\right]$ so it appears that $\lambda_{\mathrm{A}}$ does not greatly exceed $\lambda_{\mathrm{E}}$. This implies that in this low temperature range, the dominant path of conversion is between the $m= \pm 2$ and $m=0$ levels.

The $\mathrm{E}^{\mathrm{a}}$ to $\mathrm{E}^{\mathrm{b}}$ transitions may be dealt with in a similar way, but since they are degenerate (neglecting here the nuclear Zeeman splitting) the denominator of equation (16) is 
1 in this case. There are two complicating factors though which arise because the only dipolar matrix elements connecting $\mathrm{E}^{\mathrm{a}}$ and $\mathrm{E}^{\mathrm{b}}$ states are due to interactions between the methyl group and other protons. This means $(a)$ the change in the dipolar energy is difficult to calculate $(b)$ the matrix elements only connect states whose $m$ values differ by 1 , so that rotational state mixing induced by the small hindering barrier is necessary in order to account for transitions between degenerate levels. Neither of these affects the temperature dependence, but our expression for $U$ includes an unknown constant $K$. The contribution to $U$ due to transitions between the $m= \pm 1$ states is

$$
\left(Z_{\mathrm{A}}+Z_{\mathrm{E}}\right)^{-1} K \tau_{1} \exp \left(-W_{10} /(k \theta)\right)
$$

The contribution due to transitions between the $m= \pm 2$ levels is smaller at low temperatures because of the shorter lifetimes, and the smaller Boltzmann factor. The transitions between $\mathrm{A}$ and $\mathrm{E}$ states contribute to $U$ and $V$ about equally, so we write

$$
U=V+\left(Z_{\mathrm{A}}+Z_{\mathrm{E}}\right)^{-1} K \lambda_{\mathrm{E}}^{-1} \exp \left[\left(W_{20}-2 W_{10}\right) /(k \theta)\right]
$$

The curves of figure 3 have been calculated using the values $\lambda_{\mathrm{E}}=7 \times 10^{11} \mathrm{~s}^{-1}, \mathrm{~K}=$ $4.4 \times 10^{9} \mathrm{~s}^{-2}$, and $\lambda_{\mathrm{A}}$ is neglected.

\section{The temperature dependence of $T_{1}$}

Since the fastest transitions at low temperatures are from $m=1$ to -1 , these are expected to dominate spin-lattice relaxation. The temperature dependence of $T_{1}^{-1}$ is therefore expected to be similar to (19) except that at sufficiently low temperatures when $\lambda_{E} \exp \left(-W_{21} /(k \theta)\right) \sim \omega_{0}$, the nuclear Larmor frequency, one expects a minimum in $T_{1}$, and the temperature dependence of the thermal capacity of the nuclear Zeeman reservoir must also be taken into account. The chief reason why $T_{1}^{-1}$ is less than $U$ arises from the large heat capacity of the A type methyl groups. Since this is proportional to $I(I+1)$ where $I$ is the total nuclear spin quantum number, it is five times larger than for $E$ type groups. Only the E type molecules make the fast relaxing transitions, so the observed rate constant for the relaxation of the whole nuclear Zeeman reservoir contains a factor $Z_{\mathrm{E}} /\left(Z_{\mathrm{E}}+5 Z_{\mathrm{A}}\right)$ where the corresponding factor for $U$ would be closer to $Z_{\mathrm{E}} /\left(Z_{\mathrm{E}}+Z_{\mathrm{A}}\right)$. Thus we write for $T_{1}^{-1}$ the following approximate expression:

$T_{1}^{-1}=V+K\left(Z_{\mathrm{E}}+5 Z_{\mathrm{A}}\right)^{-1} \lambda_{\mathrm{E}} \exp \left[-W_{20} /(k \theta)\right]\left\{\lambda_{\mathrm{E}}^{2} \exp \left[-2 W_{21} /(k \theta)\right]+\omega_{0}^{2}\right\}^{-1}$.

In fact there should be a term in $\left(2 \omega_{0}\right)^{2}$ as well as one in $\omega_{0}^{2}$ to include double spin flips as well as single ones, but the treatment is approximate and $(20)$ is sufficient to discuss the experimental data. Above $20 \mathrm{~K}, \mathrm{E}^{\mathrm{a}}$ to $\mathrm{E}^{\mathrm{b}}$ transitions become relatively unimportant, but higher levels than those shown in figure 2 should be taken into account. Equation (20) is compared with experimental data in figure 3. A minimum in $T_{1}$ has been found (Zweers et al 1974) though it is somewhat broader, shallower and less frequency sensitive than (20) predicts. This discrepancy may reflect the inadequacy of a treatment which regards the spin-independent transitions as random processes and merits further study.

\section{The modified Haupt experiment}

The experiments which we have undertaken have two objectives. The first is to test the validity of the coupled equations (11) in a more rigorous way than simply by means of (1). The second is to extend the measurements of the faster rate constant $b$ to lower temperatures. Values of $b$ deduced from the Haupt experiment are not very accurate since they 
depend on measurements made immediately after the temperature jump, and before one can be sure that the sample temperature is uniform. This fact was recognized by Haupt (1973) who gives a curve but no experimental points for this parameter. Moreover this curve flattens around $0.02 \mathrm{~s}^{-1}$, probably indicating the upper limit due to the thermal time constants associated with the temperature jump.

Both objectives are achieved by a small modification of the Haupt experiment. By combining the initial temperature jump with one or more bursts of RF pulses at later times, one expects to observe a dipolar polarization of the same form as equation (1), though with the time origin now defined by the pulse burst, and with a value of $C$ determined by the value of $\sigma$ at that time. We check that the form of the dipolar polarization curve is independent of the time of the pulse burst $t_{0}$, and measure the rate constants $a$ and

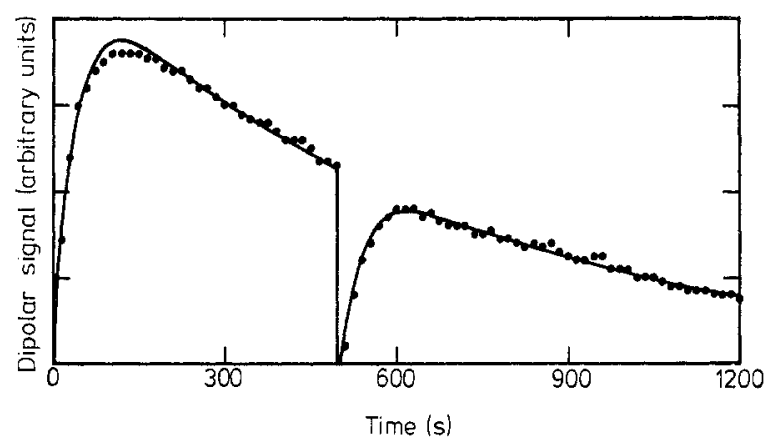

Figure 4. The modified Haupt experiment. The time evolution of the dipolar polarization is shown following a temperature jump from $60 \mathrm{~K}$ to $10.2 \mathrm{~K}$. At $480 \mathrm{~s}$ the dipolar polarization was destroyed by a series of pulses. The curves have the form of equation (1).

$b$ long after the initial temperature jump. Within experimental error the rate constants are independent of $t_{0}$ and $C$ is proportional to $\exp \left(-a t_{0}\right)$. Figure 4 shows the results of a typical experiment. The rate constant $b$ can easily be measured down to $4.2 \mathrm{~K}$. Our measurements of $a$ are in good agreement with those of Haupt as shown in figure 3.

\section{Thermal study of symmetry conversion}

Since $\sigma$ declines according to $\sigma=\sigma_{0} \exp (-a t)$, energy is therefore lost to the lattice at a similar rate. As a check on the theoretical model which in no way depends on the nuclear spins, we have observed the effect of this slow release of energy on the evaporation rate of a helium reservoir in which the sample was immersed. A large sample (28 g) of 4methylpyridine, contained in re-entrant glass tubes to provide good thermal contact with the liquid helium, was suddenly immersed in a helium reservoir, which was itself surrounded by a helium-cooled thermal shield. The subsequent evaporation rate of the helium was measured over a period of several hours by collecting the helium gas. The initial fast rate of evaporation due to the cooling of the lattice vibrations declined after about $15 \mathrm{~min}$, to be followed by a slow exponential decline in the cooling rate with a time constant of $3800 \mathrm{~s}$. The data are shown in figure 5 and compared with the results of two other experiments. An identical experiment carried out with a sample of benzene showed no similar long-term contribution to the cooling rate, which became essentially inde- 
pendent of time after the first $15 \mathrm{~min}$. The dipolar polarization induced by the Haupt effect declines at $4 \cdot 2 \mathrm{~K}$ with just this time constant of $3800 \mathrm{~s}$ and this is also shown in figure 5 .

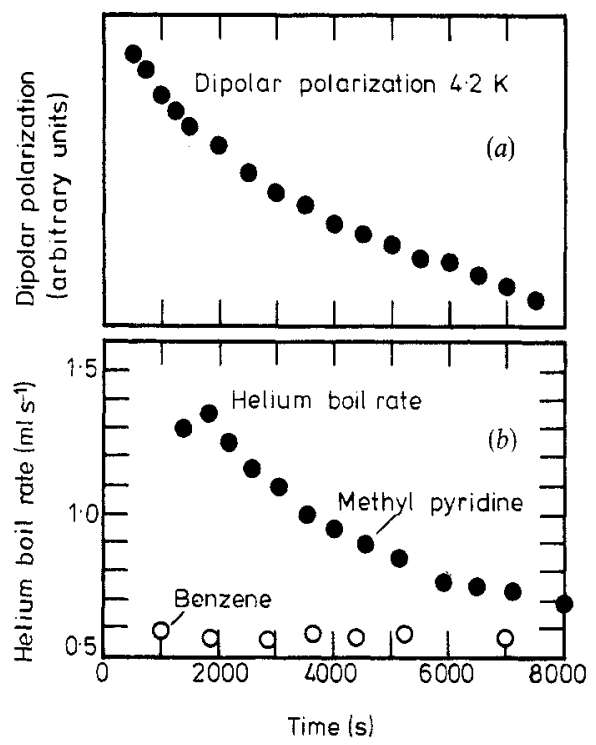

Figure 5. Thermal observation of the nuclear spin symmetry conversion at $4.2 \mathrm{~K}$. The rate of evaporation of helium is shown in (a) following the sudden immersion of a sample. For 4 methylpyridine the evaporation rate has an exponential component having the same time constant as the decay of the dipolar polarization $(b)$.

From the thermal data it is possible to make a rough estimate of the total amount of energy released to the lattice as a result of the spin symmetry conversion, and compare it with the theoretical estimate. The latter is $\frac{1}{2} W_{10} N \sigma_{0}$ since at $4.2 \mathrm{~K}$ only the lowest $\mathrm{E}$ and $\mathrm{A}$ levels are significantly populated. For a temperature jump from high temperature to $4.2 \mathrm{~K} \sigma_{0}=0 \cdot 6$, so one expects a total energy transfer of $1.8 R \mathrm{~J} \mathrm{~mole}{ }^{-1}$, where $R$ is the gas constant. Thus for a sample of 0.31 mole an energy release of $4.6 \mathrm{~J}$ is obtained.

The observed evaporation rate shown in figure 5 is well described by

$$
\mathrm{d} v / \mathrm{d} t=[5.43+0.012 t+12.38 \exp (-t / 3800)] \times 10^{-4} \text { litre }^{-1}
$$

If the exponential term is identified with the conversion and integrated between $1500 \mathrm{~s}$ and infinity, (the energy release due to conversion only beginning when the lattice has cooled well below $20 \mathrm{~K}$, a volume of 3.05 litre (at STP) of helium gas is obtained due to conversion. Taking the latent heat of helium to be $88 \mathrm{~J} \mathrm{~mole}^{-1}$, an observed energy release of $12.0 \mathrm{~J}$ is obtained, more than twice the theoretical value. It is difficult to account for the surplus energy and further experiments are planned to investigate it more thoroughly.

\section{Conclusion}

A rather simple model for the Haupt effect has explained nearly all the main experimental features. The dipolar polarization is due to a correlation between dipolar energy shifts and 
transition probabilities for $\mathrm{A}$ to $\mathrm{E}$ transitions due to the intramethyl group dipole-dipole interaction. A net transfer from $\mathrm{E}$ to $\mathrm{A}$ species occurs on cooling because the partition functions for the two species become unequal below $50 \mathrm{~K}$. (This rather high temperature is associated with the almost free rotation of the group; for more strongly hindred groups the corresponding temperature would be low and this accounts for the fact that the effect has not been observed in other materials). While $A$ to $E$ transitions generate dipolar polarization, $E^{a}$ to $E^{b}$ transitions cause its decay. Because these latter transitions occur between degenerate levels they become more efficient as the temperature falls while the polarizing transitions become less efficient. This explains why the Haupt effect is most marked between 10 and $20 \mathrm{~K}$. Some details, notably the frequency independence of the spin-lattice relaxation rate at low temperatures are not in agreement with the theory.

\section{Acknowledgments}

The debt which this work owes to the careful experiments and penetrating insight of Dr Haupt's papers is clear. One of us (SC) would also like to acknowledge several stimulating discussions with him. We are grateful for the advice of Dr M Heath and the practical assistance of Mr V Conway in connection with the thermal experiments. During the course of this work, JWH held a Science Research Council senior visiting fellowship, and PB a Canadian National Research Council Fellowship. The work forms part of a programme supported by a Science Research Council grant.

\section{References}

Alefeld B, Kollmar A and Dasannacharya B 1975 J. Chem. Phys. 63 4415-7

Clough S 1976 J. Phys. C: Solid St. Phys. 9 1553-64

Clough S and Hill J R 1974 Phys. Lett. 49A 461-2

Emid S, Wind R A and Clough S 1974 Phys. Rev. Lett. 33 769-71

Goldman M 1970 Spin Temperature and Nuclear Magnetic Resonance in Solids (Oxford: Oxford University Press)

Haupt J 1972 Phys. Lett. 38A 389-90

— 1973 Z. Naturf. 28a 98-118

Jeener J 1968 Adv. Mag. Res. 3 206-316

Look D C and Lowe I J 1966 J. Chem. Phys. 44 2995-3000

Zweers A E, Brom H B and Huiskamp W J 1974 Phys. Lett. 47 A 347-8 